\title{
Immunomodulating Properties of Two Synthetic Adjuvants: Dependence upon Type of Antigen, Dose, and Time of Administration
}

\author{
LuUk A. Th. Hilgers, Harm Snippe, Margriet Jansze, \\ AND JAN M. N. WILLERS \\ Laboratory of Microbiology, State University of Utrecht, 3511 GG Utrecht, The Netherlands
}

\author{
Received December 15, 1983; accepted February 11, 1984
}

\begin{abstract}
The effects of two synthetic adjuvants on the antibody response to sheep red blood cells (SRBC) as a thymus dependent (TD) antigen and to dinitropheny $1_{59}$-Ficoll as a thymus-independent (TI-1) antigen were investigated in mice. Both dimethyldioctadecylammonium bromide (DDA) and dextran sulfate (DXS) augmented the humoral response to SRBC but not to dinitrophenyl ${ }_{99}-$ Ficoll if injected simultaneously with antigen. Dose-response curves of both antigen and adjuvant revealed that DXS compared to DDA is a more effective adjuvant for the induction of a humoral response to SRBC. Intraperitoneal injection of DDA or DXS evoked a sequence of distinct immune responsive states in mice, measured by the capacity to develop an anti-SRBC response. A short immune-potentiating period $(<6 \mathrm{hr})$ is followed by a suppressive, second immunepotentiating state. The immune suppressive state lasted for a period of about 8 days and was restricted to TD-antigens. Suppression could be totally overridden by injection of DDA or DXS simultaneously with antigen, suggesting that the suppressive state was reversibic. The kinetics of the observed alteration of the immune response by DDA and DXS were very similar. It is concluded that differences in the modulation of the immune response by DDA and DXS are limited to the initial state. Long-term effects like the induction of a succession of distinct immune responsive states, are more or less similar for both adjuvants. Possible mechanisms by which these immunomodulators interfere with the immune system are discussed.
\end{abstract}

\section{INTRODUCTION}

Many adjuvants which are supposed to stimulate the immune response instead cause immunosuppression. Several adjuvants such as phytohemagglutinin, Bacillus Calmette-Guerin (BCG), Bordetella pertussis, Freund's complete adjuvant (FCA), and dextran sulfate (DXS) have been shown to suppress as well as to stimulate cellmediated immune responses $(1,2)$. The kind of effect following administration of adjuvant depended on route, dosage, time of administration, and antigen used (2).

In this study two synthetic adjuvants greatly differing in physicochemical properties and biological activities were compared on their immunomodulating capacities. Dimethyldioctadecylammonium bromide (DDA) is a positively charged lipophilic quarternary amine with a low molecular weight (MW 631) and is almost insoluble in water. On simultaneous injection DDA may alter the properties of an antigen by direct attachment (3) or formation of complexes (4). Both delayed-type hypersensitivity $(5-7)$ and antibody formation $(8,9)$ can be enhanced by DDA. Dextran sulfate is a 
negatively charged polyanion with a high molecular weight (MW 500,000) and is soluble in water. Humoral responses can be stimulated upon the use of DXS (10, 11 ), while cell-mediated immune responses can be both stimulated $(12,13)$ and suppressed (14).

In the present study the effects of DDA and DXS on the humoral response to a thymus-dependent (TD) antigen and thymus-independent antigen were compared. The time interval between the administration of adjuvant and antigen greatly determined the effect of these agents on the antibody formation.

\section{MATERIALS AND METHODS}

Animals. Inbred BALB/c mice were raised and maintained in the Laboratory of Microbiology, State University, Utrecht, The Netherlands. Female mice were used at an age of about 10 weeks (weight approximately $20 \mathrm{~g}$ ).

Administration of $D D A, D X S$, sheep red blood cells (SRBC), and dinitrophenyl $\left(D N P^{s}\right)$-Ficoll. Mice were injected intraperitoneally (ip) with $200 \mathrm{nmol}$ DDA (Kodak Eastman, Rochester, N.Y.) or $1 \mathrm{nmol}$ DXS (Pharmacia, Uppsala, Sweden) in $0.5 \mathrm{ml}$ saline, unless stated otherwise.

Mice were immunized by intraperitoneal injection of $2 \times 10^{7} \mathrm{SRBC}_{\text {or }} 3 \mu \mathrm{g} \mathrm{DNP}_{59-}$ Ficoll. The latter antigen was prepared according to the procedure of Inman (15).

Hemolytic plaque assay. The number of direct plaque-forming cells (PFC) in spleen cell suspensions was determined by a modification of the Jerne hemolytic plaque assay as described by Merchant and Inman (16). SRBC or DNP-SRBC, prepared according to the procedure of Inman et al. (17), were used as indicator erythrocytes.

Antibody detection. Blood was withdrawn from the retroorbital venous plexus. The presence of antibodies was determined with a hemagglutination assay. Twofold dilutions of antiserum in saline (supplemented with $1 \%$ normal rabbit serum) were prepared in microtiter plates (Greiner, Nürtingen, West Germany). Fifty microliters of a suspension of $0.5 \%$ SRBC was added to $50 \mu \mathrm{l}$ of the diluted antiserum. Hemagglutination was determined after $4 \mathrm{hr}$. The reciprocal value of the endpoint of hemagglutination was taken as the titer.

Statistical analysis. Results are expressed as the arithmetic means of $n$ independent observations \pm the standard error of the mean (SEM). Student's $t$ test was performed to analyze the statistical significance of the results. $P$ values over 0.05 are considered to be not significant.

\section{RESULTS}

Effects of DDA and DXS on the Humoral Response to a Thymus-Dependent Antigen and Thymus-Independent Antigen

Groups of mice were injected ip with either saline, $200 \mathrm{nmol}$ DDA, or $1 \mathrm{nmol}$ DXS and with $2 \times 10^{7}$ SRBC or $3 \mu \mathrm{g} \mathrm{DNP}_{59}-$ Ficoll, and the numbers of PFC in the spleen were determined 5 days later. Both DDA and DXS significantly enhanced the response to $\mathrm{SRBC}$, but not that to $\mathrm{DNP}_{59}-$ Ficoll (Fig. 1). The response to SRBC was increased three-to fourfold (DDA) and five- to sixfold (DXS). Dose-response curves of the adjuvants (Fig. 2) with two different doses of antigen revealed that at $200 \mathrm{nmol}$ DDA a maximal effect on the response to SRBC was reached (Fig. 2). A low dose of DDA $(50 \mathrm{nmol})$ did not affect the humoral response as compared to untreated controls. 


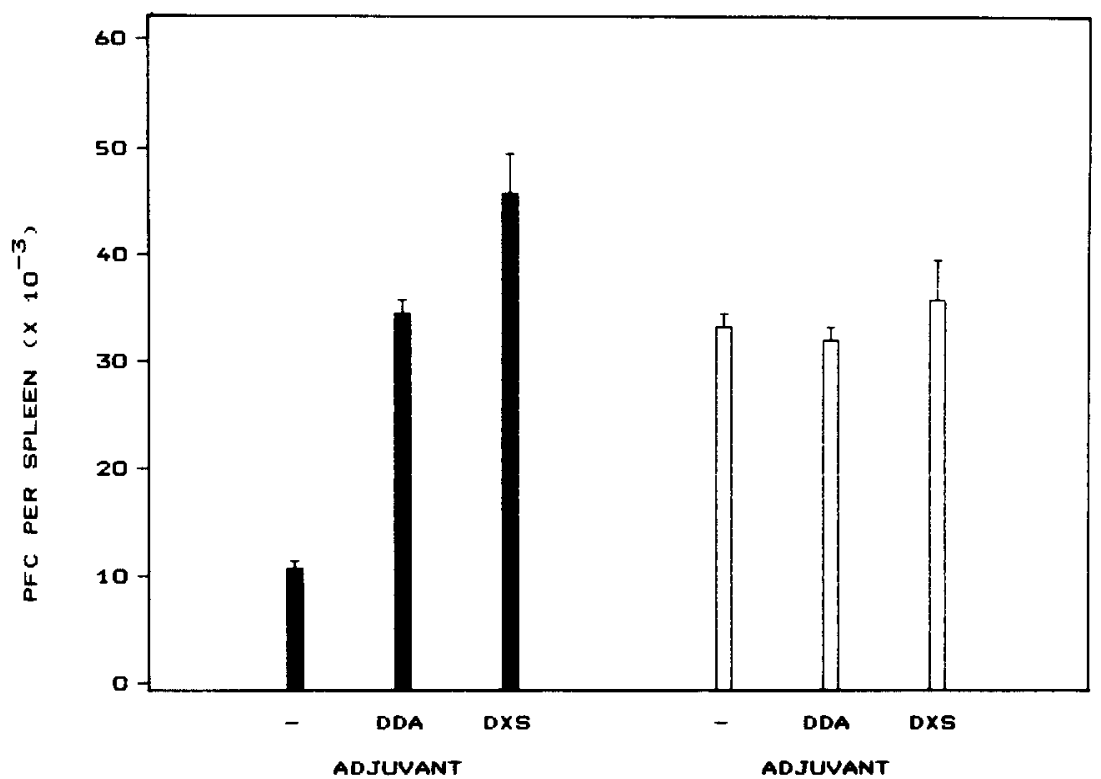

FIG. 1. Effects of DDA and DXS on the humoral response to SRBC (black columns) and DNP-Ficoll (white columns). The number of anti-SRBC and anti-DNP PFC in the spleen were measured 5 days after the ip administration of adjuvant and antigen $(n=5)$. Vertical bars indicate SEM.

In contrast to DDA, DXS evoked a dose-dependent increase of the response to SRBC (Fig. 2). The optimal dose of DXS seemed to be above $4 \mathrm{nmol}$. A 20-fold increase in the number of anti-SR BC. PFC compared to untreated controls was observed. However, two out of five mice died after the injection of $4 \mathrm{nmol}$ DXS and spleens of the survivors were markedly dilated, as measured by the total number of cells per spleen (data nol shown).
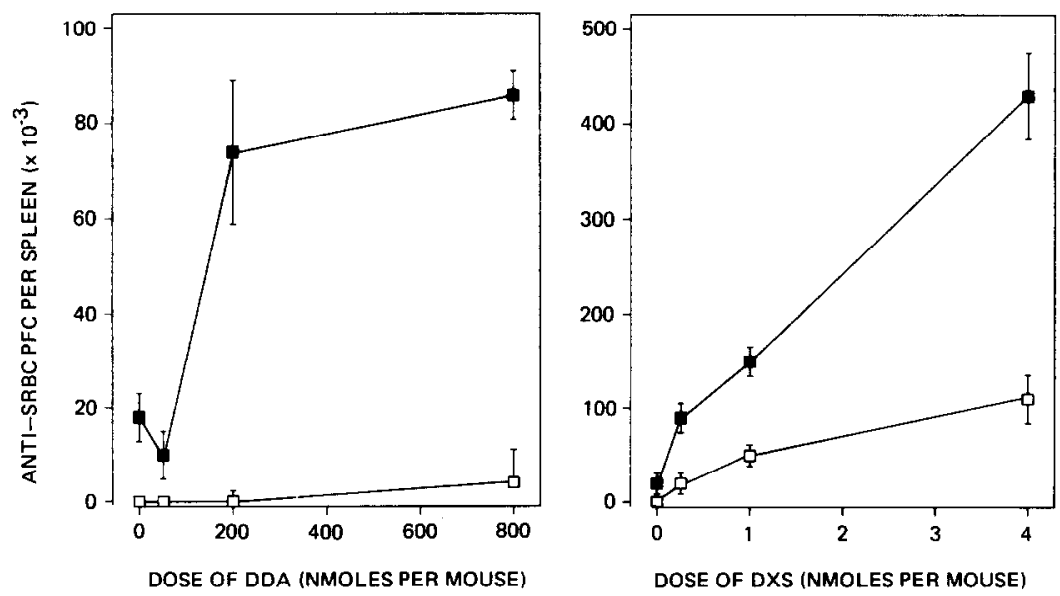

FIG. 2. Dose dependence of the effect of adjuvant on the humoral response to SRBC. Groups of mice $(n=5)$ were immunized ip with $10^{6}$ SRBC ( $\square$ ) or $2 \times 10^{7}$ SRBC ( $\square$ ) with several doses of DDA (left panel) or DXS (right panel). The numbers of PFC in the spleen were measured 5 days after immunization. Vertical bars indicate SEM. 
Dose-response curves for SRBC (Fig. 3) show distinct effects of DDA and DXS on the anti-SRBC response. DDA increased significantly the response to $5 \times 10^{6}$ and $2 \times 10^{7}$ SRBC, but failed to induce or enhance the response to smaller $\left(1.2 \times 10^{6}\right)$ and larger $\left(8 \times 10^{7}\right)$ doses of SRBC, respectively.

A larger dose of DDA $(800 \mathrm{nmol})$ was also unable to promote a humoral response to the low dose of $1.2 \times 10^{6} \mathrm{SRBC}$ (Fig. 2). DXS, however, enhanced the humoral response to a wide dose range of SRBC. Small doses of SRBC $\left(3 \times 10^{5}\right.$ to $\left.5 \times 10^{6}\right)$ became immunogenic upon addition of $1 \mathrm{nmol}$ DXS. The response to doses of SRBC, which already evoked a detectable humoral response without adjuvant, were significantly enhanced by $1 \mathrm{nmol}$ DXS.

\section{Effects of DDA and DXS Injected Prior to the Antigen}

Groups of five mice were injected ip with a single dose of $200 \mathrm{nmol}$ DDA or 1 nmol DXS. Simultaneously with the adjuvants or $6 \mathrm{hr}$ to 16 days later, all mice were immunized with $2 \times 10^{7} \mathrm{SRBC}$ or $3 \mu \mathrm{g} \mathrm{DNP}{ }_{59}-$ Ficoll. The humoral responses to SRBC and to DNP were measured at Day 5. Injection of DDA or DXS $6 \mathrm{hr}$ prior to the antigen enhanced the response to SRBC (Fig. 4). In contrast, administration of DDA or DXS 1 to 4 days before immunization resulted in an anti-SRBC response which was below the level of immunized controls which received no adjuvant.

Immunization with SRBC 12 to 16 days after the administration of DDA or DXS resulted in an enhanced anti-SRBC response. The optimal anti-SRBC response in mice treated with DDA was obtained if the adjuvant was injected 12 days before the antigen. DXS was most effective if it was injected simultaneously with or $6 \mathrm{hr}$ prior to the antigen. The response to the $\mathrm{TI}$ antigen $\mathrm{DNP}_{59}$-Ficoll was not affected by the

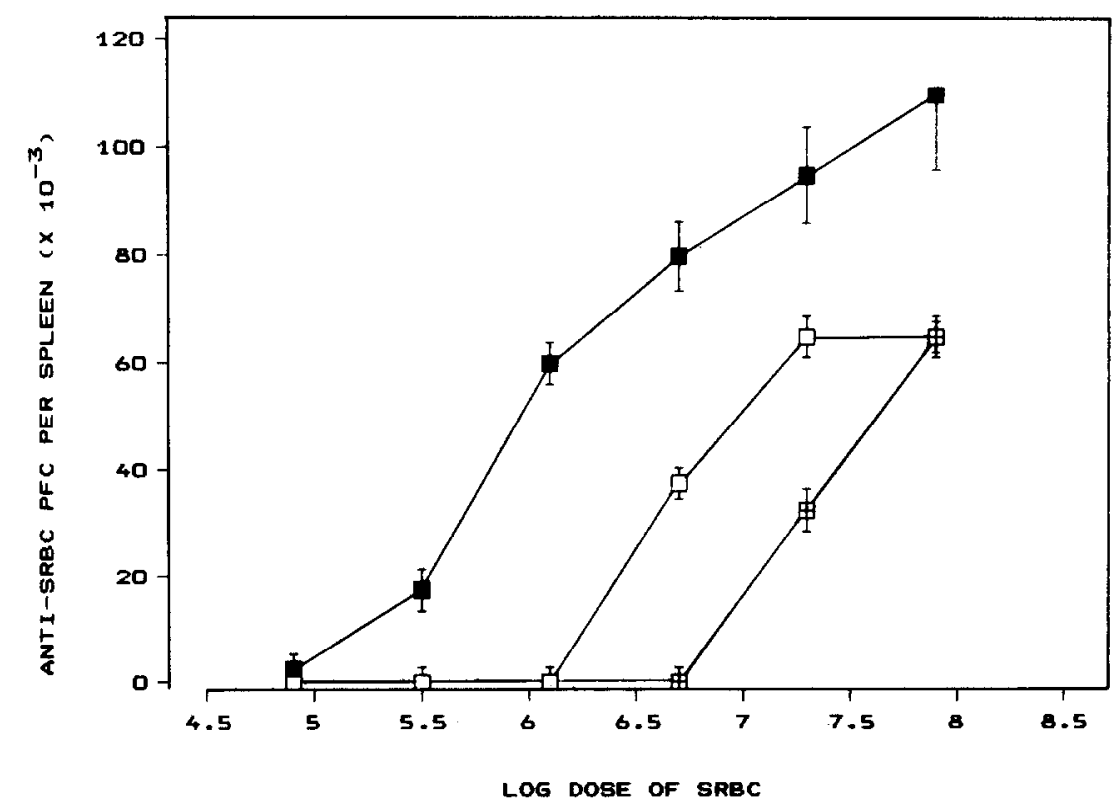

Fig. 3. Dose dependence of the response to SRBC with and without adjuvant. Groups of mice $(n=5)$ were immunized ip with several doses of SRBC with DDA ( $(\varpi)$ or DXS ( $\square$ ) or without $(由)$ adjuvant. The numbers of PFC in the spleen were measured 5 days after immunization. Vertical bars indicate SEM. 

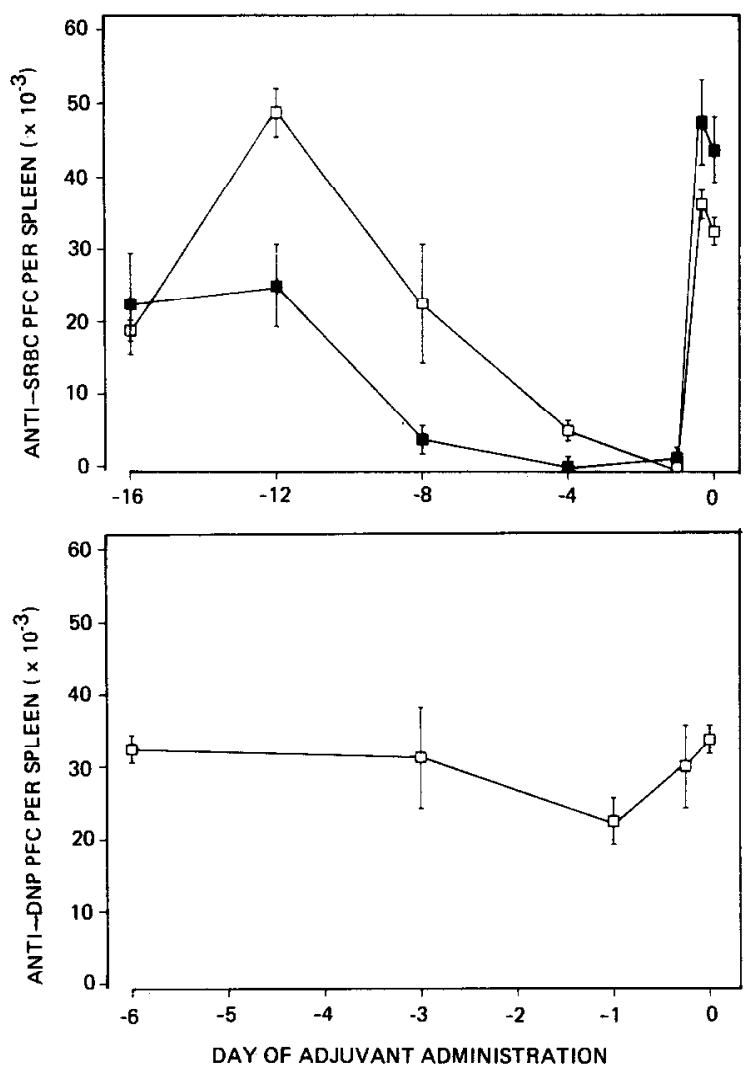

FIG. 4. Effects of the interval between the administration of adjuvant and antigen on the humoral response to SRBC (upper panel) and DNP ${ }_{59}-$ Ficoll (lower panel). Mice $(n=5)$ were injected ip with DDA ( $)$ or DXS ( $\square)$. After different intervals mice were injected ip with $2 \times 10^{7} \mathrm{SRBC}$ or $3 \mu \mathrm{g} \mathrm{DNP}$-Ficoll and the numbers of PFC in the spleen were measured 5 days later. The number of PFC per spleen (mean \pm SEM) in controls $(n=5)$, which received SRBC or DNP $_{59}$-Ficoll without adjuvant, was $10 \times 10^{3} \pm 0.8 \times 10^{3}$ or $35 \times 10^{3} \pm 4 \times 10^{3}$, respectively. Vertical bars indicate SEM.

injection of DDA prior to the antigen, with the exception of injection of the adjuvant 1 day before.

\section{Characterization of the Immune Suppressive State}

As shown in Fig. 4, ip injection of DDA or DXS 1 or 4 days prior to immunization with SRBC resulted in a remarkable reduction of the number of anti-SRBC PFC in the spleen 5 days after immunization (Day 5). This suppressive state was further investigated. The number of PFC in the spleen of mice pretreated with DDA or DXS on Day -1 or -4 remained low from 4 to 7 days after immunization (data not shown). The titer of anti-SRBC antibodies in the blood of these mice was measured on Days 5 and 7. Administration of DDA 1 day prior to the antigen resulted in undetectable antibody titers, while DDA given 4 days and DXS given 1 or 4 days before immunization resulted in low antibody titers (Fig. 5).

The immune suppressive state could be partly counteracted by elevating the SRBC dose, but the response remained below that of untreated controls (data not shown). 


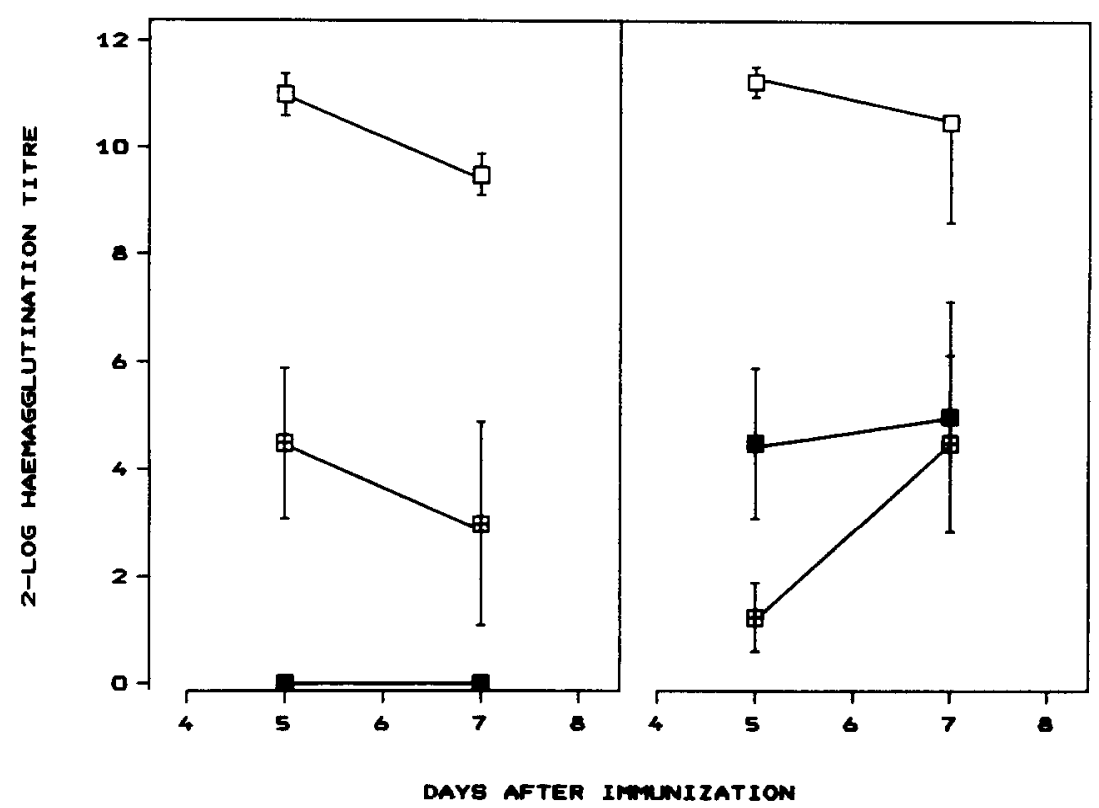

FIG. 5. The anti-SRBC antibodies in the blood several days after immunization of mice pretreated with DDA (left panel) or DXS (right panel). Groups of mice $(n=5)$ were ip injected with DDA or DXS $0(\square)$,

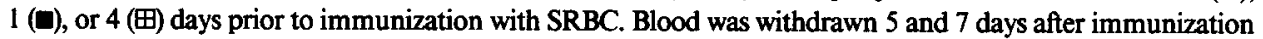
and the anti-SRBC antibody titer was determined. Vertical bars indicate SEM.

However, DDA- and DXS-induced immunosuppression could be totally overridden by the addition of DDA or DXS to the antigen. Administration of DDA or DXS on Day -4, followed by immunization with SRBC with either DDA or DXS on Day 0 , resulted in an enhanced humoral response (Fig. 6). The response in pretreated mice was as high as that in mice which received antigen and adjuvant simultaneously but were not pretreated with DDA or DXS (compare Figs. 6 and 1).

\section{DISCUSSION}

The results obtained demonstrate similarities and differences in the effect of two synthetic adjuvants (DDA and DXS) on the humoral immune response. The effects of these agents greatly depend on the time interval between the administration of adjuvant and antigen. The immunomodulating effects of DDA and DXS were restricted to thymus-dependent antigens, as the response to SRBC was affected but not the response to $\mathrm{DNP}_{59}$-Ficoll. This is in agreement with the general concept that most adjuvants act on macrophages and on T-cell subsets (18).

Both DDA and DXS augmented the fifth day humoral response to a normal immunogenic dose of $2 \times 10^{7} \mathrm{SRBC}$. DXS stimulated the response to low doses of SRBC $\left(10^{5}-10^{6}\right)$, but DDA was ineffective in this respect. Maximal augmentation of the response by DXS was greater than by DDA and was reached when $4 \mathrm{nmol}$ DXS was injected ip simultaneously with $2 \times 10^{7} \mathrm{SRBC}$. Dose-response curves revealed that DXS was effective over wider ranges of dose of both adjuvant (Fig. 2) and antigen (Fig. 3) than DDA.

Intraperitoneal administration of DDA or DXS evoked a sequence of immune responsive states. Depending on the time interval between adjuvant and antigen 


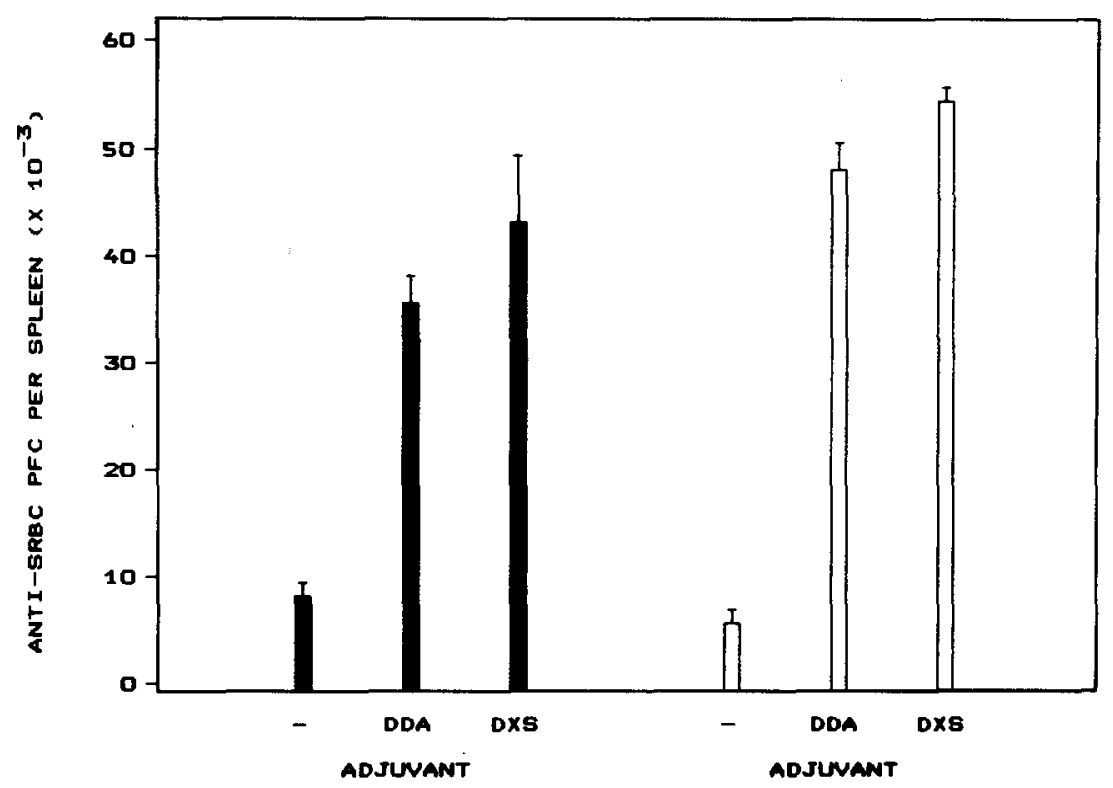

FIG. 6. Effect of pretreatment with adjuvants on the humoral response to SRBC mixed with adjuvant. Groups of mice ( $n=5$ ) were injected ip with DDA (black columns) or DXS (white columns) on Day -4 . On Day 0 the mice were immunized with SRBC alone or mixed with DDA or DXS. The numbers of antiSRBC PFC in the spleen were measured on Day 5. Vertical bars indicate SEM.

injections, the humoral anti-SRBC response was enhanced or suppressed. After an immune-potentiating period of at least $6 \mathrm{hr}$ an immune suppressive state of 4 to 8 days was followed by a second immune-potentiating state of at least 8 days. The patterns of alternating immune-potentiating and suppressive states after injection of DDA and DXS were very similar.

Time-dependent immunomodulation has been (partially) described for some other agents. Proteose peptone (19), Corynebacterium parvum (19, 20), and adriamycin (21) all induced an immune suppressive state to SRBC, which lasted for 4-16 days. Peritoneal macrophages obtained from these mice displayed a stronger ability to phagocytize and degrade SRBC than resident macrophages (19). In the immune response, macrophages play a number of different roles, such as scavenger cells, antigen-presenting cells $(22,23)$, and regulators of the immune response by release of several mediators. Prostaglandins (24-27), complement components (28), oxygen intermediates (29), thymidine (30), and exoenzyme (31) released from macrophages are able to suppress the immune response. Data presented by others (19) and data from our studies (L. A. Th. Hilgers et al., manuscript in preparation) indicate that suppression of the immune response is due mainly to the increased activity of macrophage as scavenger cells, resulting in a reduction of effective antigenic stimulation.

The suppressive state induced by either DDA or DXS alone could be easily overridden by a second injection of an adjuvant simultaneously with the antigen (Fig. 6). This suggests that the suppressive state is reversible. If suppression is due to increased macrophage activity, addition of adjuvant to the antigen may depress this activity, resulting in prolonged persistence of the antigen. The persistence of SRBC will result in an increased humoral response to the antigen. This suggests that an 
important mode of action of these adjuvants may be prolongation of the persistence of the antigen by depressing clearance and breakdown by macrophages. Other investigators have affirmed that these agents are capable of interacting with macrophages. DDA affected functional properties of macrophages as measured by decreased phagocytosis of yeast cells, decreased acridine orange uptake by lysosomes, and decreased spreading (32). DXS induced lysosomal enzyme release by macrophages in vitro (33) and reduced the nonspecific resistance to Salmonella typhimurium in vivo as a consequence of impaired macrophage activity (34).

Differences between DDA and DXS may be due to minor effects of these agents on the immune system or differences in the way they affect macrophages. DDA is capable of forming complexes with SRBC (4), which may result in an altered uptake or processing of the antigen by macrophages. Intraperitoneal injection of DDA induced a sixfold increase in the number of peritoneal cells at $24 \mathrm{hr}$, in contrast to DXS which induced only a twofold increase (L. A. Th. Hilgers et al., manuscript in preparation). This may contribute to the suppression of the immune response as the clearance capacity of the total of peritoneal cell population is increased: immunization with SRBC 1 day after ip injection of DDA resulted in a complete absence of circulating antibodies (Fig. 5). DXS was shown to be a potent polyclonal B-cell activator in vitro (35-37), but the contribution of this property to immunomodulating activity in vivo may be negligible compared to the effect on macrophages. This assumption is supported by the observation that the immune response to a thymus-independent antigen is not enhanced by DXS (Fig. 1).

In conclusion, similarities and differences between the two synthetic adjuvants, DDA and DXS, were observed. Both adjuvants augmented the humoral response to a TD antigen (SRBC) but not to a $\mathrm{TI}$ antigen ( $\mathrm{DNP}_{59}$-Ficoll), when adjuvant and antigen were injected simultaneously. A sequence of suppressive and immune-potentiating states was induced by ip injection of DDA or DXS. When adjuvant and antigen were injected simultaneously differences between DDA and DXS were observed with respect to the effective range of adjuvant and antigen doses.

\section{ACKNOWLEDGMENTS}

This work was supported by the Division of Health Research TNO (HGO-TNO) in cooperation with the Foundation for Medical Research Fungo (Project 13-27-70).

\section{REFERENCES}

1. Jollés, P., and Paraf, A., In "Molecular Biology, Biochemistry and Biophysics" (A. Kleinzelles, G. F. Springer, and H. G. Whitmann, Eds.), Vol. 13, p. 1. Springer, New York, 1973.

2. Schwab, J., Bacteriol. Rev. 39, 121, 1975.

3. Dailey, M. D., and Hunter, R. L., J. Immunol. 112, 1526, 1974.

4. Baechtel, F. S., and Prager, M. D., Cancer Res. 42, 4959, 1982.

5. Chiba, J., and Egashira, Y., Japan. J. Med. Sci. Biol. 31, 361, 1978.

6. Snippe, H., Belder, M., and Willers, J. M. N., Immunology 34, 363, 1977.

7. Snippe, H., Johannesen, L., Lizzio, E., and Merchant, B., Immunology 39, 399, 1980.

8. Gall, D., Immunology 11, 369, 1966.

9. Gorden, W. C., and Prager, M. D., Cell. Immunol. 49, 329, 1980.

10. Diamantstein, T., Wagner, B., Beyse, I., Odenwald, M. V., and Schulz, G., Eur. J. Immunol. 1, 340, 1971.

11. Bradfield, J. W. B., Soumahi, R. L., and Addison, I. E., Immunology 26, 383, 1974.

12. Kishima, M., Kuniyasu, C., and Nakagawa, M., Infect. Immun. 39, 823, 1983. 
13. McCarthy, R. E., Arnold, L. W., and Babcock, G. F., Immunology 32, 963, 1977.

14. Babcock, G. F., and McCarthy, R. E., Immunology 33, 925, 1977.

15. Inman, J. K., J. Immunol. 114, 704, 1975.

16. Merchant, B., and Inman, J. K., J. Exp. Med. 145, 372, 1977.

17. Inman, J. K., Merchant, B., Claflin, L., and Tacey, S. E., Immunochemistry 10, 165, 1973.

18. Bomford, R., Int. J. Tissue Reac. 4, $201,1982$.

19. Yoshikai, Y., Maike, S., Sano, M., and Nomoto, K., Cell. Immunol. 77, 266, 1983.

20. Ghaffar, A., and Sigel, M. M., Immunology 35, 685, 1978.

21. Ehrke, M. J., Tomazic, V., Kyoyama, K., Cohen, S. A., and Mihich, E., Int. J. Immunopharmacol. 5, 43, 1983.

22. Unanue, E. R., In "Advances in Immunology" (F. J. Dixon and H. G. Kunkel, Eds.), Vol. 15, p. 95. Academic Press, New York, 1972.

23. Unanue, E. R., In "Advances in Immunology" (H. G. Kunkel and F. J. Dixon, Eds.), Vol. 31, p. 1. Academic Press, New York, 1981.

24. Stenson, W. F., and Parker, C. W., J. Immunol. 125, 1, 1980.

25. Webb, D. R., and Jamieson, A. T., Cell. Immunol. 24, 45, 1976.

26. Webb, D. R., and Nowowiejski, I., Cell. Immunol. 33, 1, 1977.

27. Henney, C. S., Bourne, H. R., and Lichtenstein, L. M., J. Immunol. 108, 1526, 1972.

28. Edwang, T. G., and Befus, A. D., Immunology 51, 207, 1984.

29. Metzger, Z., Hoffeld, J. T., and Oppenheim, J. J., J. Immunol. 124, 983, 1980.

30. Stadecker, M. J., Calderon, J., Karnovsky, M. L., and Unanue, E. R., J. Immunol. 119, 1738, 1977.

31. Kung, J. Y., Brooks, S. B., Jahway, J. P., Leonald, L. L., and Tahnge, D. W., J. Exp. Med. 146, 665, 1977.

32. Bloksma, N., De Reuver, M. J., and Willers, J. M. N., Antonie van Leeuwenhoek 49, 13, 1983.

33. Schorlemmer, H. U., Burger, R., Hylton, W., and Allison, A. C., Clin. Immunol. Immunopathol. 7, 88, 1977.

34. Hof, H., Emmerling, P., Hacker, J., and Hughes, C., Ann. Immunol. (Paris) 133, 21, 1982.

35. Diamantstein, T., Rühl, H., Vogt, W., and Bochert, G., Immunology 25, 743, 1973.

36. Nariuchi, H., and Kakiuchi, T., Cell. Immunol. 61, 375, 1981.

37. Wetzel, G. D., and Kettman, J. R., Cell. Immunol. 61, 176, 1981. 